\title{
Activity Plan Template: a mediating tool for supporting learning design with robotics
}

\author{
Nikoleta Yiannoutsou ${ }^{1}$, Sofia Nikitopoulou ${ }^{1}$, Chronis Kynigos ${ }^{1}$, Ivaylo Gueorguiev ${ }^{2}$, \\ Julian Angel Fernandez ${ }^{3}$ \\ ${ }^{1}$ UoA ETL, National and Kapodistrian University of Athens, Athens, Greece \\ nyiannoutsou@ppp.uoa.gr, sophieniki@ppp.uoa.gr, \\ kynigos@ppp.uoa.gr \\ ${ }^{2}$ ESI CEE, European Software Institute Center Eastern Europe - Sofia, Bulgaria \\ ivo@esicenter.bg \\ ${ }^{3}$ ESI CEE ACIN Institute of Automation and Control, Vienna University of Technology, \\ Vienna, Austria \\ julian.angel. fernandez@tuwien.ac.at
}

\begin{abstract}
Although Robotics have been designed for education for several decades now, only recently they started being broadly used in education, formal and non formal. In this context many different technologies have emerged accompanied by relevant learning material and resources. Our observation is that the vast number of learning activities is driven by multiple "personal pedagogies" and thus it results in the fragmentation of the domain. To address this problem we discuss in the paper the construct of "activity plan template", a generic design tool that will facilitate different stakeholders (teachers, instructors, researchers) to design learning activities for different robotic toolkits. In the paper we discuss the characteristics of the activity plan template and the research process of generating such a template. Since we report work in progress, we present here the first version of the activity plan template, the construction of which is based on a set of best practices identified.
\end{abstract}

Keywords: Activity Plan Template; learning design; educational robotics

\section{Introduction}

The educational robotics landscape is vast and fragmented in and outside schools. In the last two decades, robots have started their incursion into the formal educational system. Although diverse researchers have pointed out their benefits in schools, the slow pace of their introduction is partially justified by the cost of the kits and the schools' different priorities in accessing technology. Recently, the cost of kits has 
decreased, whereas their capabilities and availability of supporting hardware and software has increased $[1,2]$. With these benefits, educational robotics kits have become more appealing to schools. In this context, various stakeholders - technology providers, teachers, academics, companies focusing on delivering educational material etc. - invest in the creation of different learning activities around robotic kits, in order to showcase their characteristics and make them attractive in and out of schools. Thus, a growing number of learning activities have emerged. These activities share common elements but they are also very diverse in that they address different aspects of Robotics as teaching and learning technology with their success lying in how well they have identified these aspects and how well they address them. This is partly due to the fact that Robotics is a technology with special characteristics when compared to other learning technologies: they are inherently multidisciplinary, which in terms of designing a learning activity might mean collaboration and immersion into different subject matters; they are extensively used in settings of formal and non formal learning and thus involving different stakeholders; their tangible dimension causes perturbations -especially in formal educational settings- which are closely related to the introduction of innovations in organizations and schools (i.e. from considering classroom orchestrations to establishing or not, connections with the curriculum etc.); they are at the heart of constructionist philosophy for teaching and learning [3]; they are relevant to new learning practices flourishing now over the internet like the maker movement, "Do It Yourself" and "Do It With Others" communities etc. With this in mind we argue that we need to take a step back from the level of specific learning activities and create a more generic design instrument i.e. an activity plan template, that: a) it will be pedagogically grounded on the particular characteristics of robotics as a teaching and learning tool b) it will be adaptable to different learning settings (formal - non-formal) c) it will afford generating different examples of learning activities for different types of kits d) it will focus on making explicit the implicit aspects of the learning environment and e) it will urge designers to think "out of the box" by reflecting its content. In the following sections we describe the theoretical background supporting the concept of activity plan template as a design instrument and the method for developing an activity plan template for teaching and learning with Robotics.

\section{Theoretical background}

In order to explain the role of a generic design instrument such as the activity plan template, in addressing the problem of fragmentation in the practice of using educational robotics for learning, we will discuss in this section the dimensions and functions of design in education.

"Everyone designs who devises courses of action aimed at changing existing situations into desired ones" [4 cited in, 5]. With this definition we aim to highlight that design is an integral part of the teaching profession. Acknowledging this dimension in teaching, and with the advent of digital technologies in schools, design based research has been implemented as an approach to orchestrate and study the 
introduction of innovation in education[6]. Furthermore, in the field of education, design has been introduced as the bridge between theory and practice [5] because design is expected to play a dual role: a) to guide practice informed by theory and b) to inform back the theory after the evaluation of the design in practice. Thus in this context design is not only an organized sequence of stages, all of which compose an orchestration of the learning process [7] but it is also a reflection and an evaluation tool.

Gueudet and Trouche [8] focusing mainly on resources and documents designed by teachers (e.g. activity or lesson plans), reveal another dimension of design as they describe it as a tool that not only expresses but also shapes the teacher's personal pedagogies, theories, beliefs, knowledge, reflections and practice. The term they use to describe this process is Documentational Genesis. A core element of this approach is instrumental theory [9] according to which the characteristics of the resources teachers select to use, shape their practice on the one hand (instrumentation) and on the other hand, the teachers' knowledge shapes the use of the resources as teachers appropriate them to fit their personal pedagogies (instrumentalization). Teacher designs, as a result of the above, intertwined, processes according to Pepin, B., Gueudet, G., \& Trouche [10], are evolving or living documents - in the sense that they are continuously renewed, changed and adapted.

Design as expressive medium for teachers and educators, can also function as an instrument for sharing, communicating, negotiating and expanding ideas within interdisciplinary environments. This property of activity plans is linked to the concept of boundary objects and boundary crossing [11]. The focus here is on the artefact (in our case activity plan) that mediates a co-design process by helping members of different disciplines to gain understanding of each other's perspectives and knowledge. Educational Robotics for STE(A)M is such an interdisciplinary environment which involves an understanding of related but different domains (i.e. Science, Technology, Engineering, Arts, Mathematics) and involves players from industry, academia and organizers of educational activities.

A problem with all these designs, especially when they involve integration of technologies, is that they are driven by a multitude of "personal pedagogies" the restrictions of which result in adapting technologies to existing practices [12]. Conole (ibid) argues that the gap between the potential of digital technologies to support learning and their implementation in practice can be bridged with a "mediating artefact" to support teacher designs. She continues claiming that such a mediating artefact should be structured according to specific pedagogic approaches and should focus on abstracting essential and transferable properties of learning activities that are not context bound. The activity plan template can play the role of the mediating artefact equipping professionals with a structured means to describe, share and shape their practices. This way we can contribute in addressing the problem of fragmentation in the learning activities regarding the use of Educational robotics. 


\section{Developing an activity plan template for Educational Robotics}

The work reported in this paper takes place in the context of the European project ER4STEM. The main objective of this project is to refine, unify and enhance current European approaches to STEM education through robotics in one open operational and conceptual framework. The development of activity plan templates contributes towards this direction as it provides a generic design instrument that identifies critical elements of teaching and learning with robotics based in theory and practice and in that contributes to the description of effective learning and teaching with robotics. The process through which we develop the activity plan templates in this project includes the following steps: We create a first draft based on a) on identifying and analyzing a set of good practices and b) previous work on activity plans that involve innovative use of technologies for teaching and learning. The next step is to use this first draft to design and implement workshops with Robotics in different educational settings and systems. During this implementation we will collect data that will allow us to evaluate, refine and re-design the activity plan template so as to be a useful and pedagogically grounded instrument for designing learning activities. In this paper we are at the first stages of our research and thus we will report on: a) a set of criteria that we developed in order to identify good practices and b) the first draft of the activity plan template.

\section{Identifying best practices}

The criteria for selecting best practices in the domain of educational robotics were formed through a bottom -up empirical process. Specifically, three researchers from different research teams of the consortium worked independently to select a set of best practices from robotics conferences, competitions, seminars and workshops organized by different institutions. This was the first phase of the selection process, which was not done in a structured way. The second phase included analysis and reflection on phase one. Specifically, the criteria were shaped by a) an analysis of the content of five examples of best practices already selected and b) elaboration of the criteria that researchers had implicitly applied during the selection of the specific best practices. Next the items that -from the analytic and the reflective process- were identified to be part of what could be considered best practice in the field of educational robotics were synthesized in one document.

The best practice selection criteria are designed to feed into the activity plans (and not map directly into them) by providing interesting and new ideas for concepts, objectives, artefacts b) orchestration c) teaching interventions and learning process d) implementation process and e) evaluation process. 


\section{Criteria}

The criteria developed for identifying best practices are divided in two categories. One category is mainly a set of prerequisites, which should be covered in order for an event or activity to be considered. The other category consists of the main criteria that identify best practice aspects of the activities.

\section{Prerequisites:}

- Topic includes concepts related to the following subjects: ScienceTechnology-Business-Engineering-Art-Mathematics or something from another discipline but related to robotics

- The activity- event shows that it has constructionist elements: i.e. it is not just a presentation of tools or predefined guidelines

- The activity- event is innovative, related to student or citizen interests

- The activity-event includes technology related to educational robotics

\section{Main Criteria}

In case that the "educational robotic event" is assessed as relevant according to the aforementioned basic pre-requisites, then the process continues with the assessment of the following parameters. Not all parameters have to be met in order for an event or activity to be considered as good practice. These parameters are defined in order for us to collect good practices with respect to different dimensions of robotics activities stemming from different sources.

\section{CONTEXT}

- Place: provides information about the space where the educational robotic activity takes place. This information is crucial to determine other aspects of the learning design such as orchestration issues, formal or non-formal settings etc. Possible examples can be school, museum, science institutions, or other educational scientific organizations

- Participants' description: provides information regarding issues such as age, number, culture, background etc. The activity is considered as good practice if it is aligned to the age of the participants, the number, the prior knowledge of the participants on a specific subject, etc.

- Theoretical framework: refers to the pedagogical approach used in implementing the educational activity e.g. DIY (Do It Yourself), DIWO (Do It With Others), Constructionism, STEM education, Design. In several cases the theoretical framework is implicit and can be inferred from the way the activity is orchestrated and designed.

\section{EDUCATIONAL ACTIVITY}

- Connection with a curriculum: This dimension provides information regarding issues of connecting the teaching of robotics to specific topics of national curricula. It is not expected to apply to all events or activities identified.

- Motivation for the activity: Provides information on what has motivated the organization of the specific activity (e.g. introducing girls to robotics, elaborating on specific STEM concepts, using art to explore robotics etc.). In identifying good practices we are looking for interesting motivations and 
the way the activity is organized to support this motivation. Special focus is given to events that are designed to motivate young people to learn STEM disciplines.

- Description of the activity: Provides information regarding the implementation of the activity. This information helps out in identifying if the activity matches the context the motivation etc. The activity description is expected to refer to issues regarding the duration, tasks, orchestration, grouping, learner interaction (i.e. where is the emphasis concerning the action, the relationships, the roles in the group and the teacher's role).

TOOLS

- Technology used- selection criteria: Provides information on the specific technology used for the implementation of the specific activity. It is considered as good practice if the educational robotic event is based on technology that follows the latest trends, it is compatible with the background of the participants, facilitates well the objectives and the motivation of the activity, it is presented in a way that it is understandable by the specific target group in the workshops and is similar to what young people are using in their everyday life e.g. mobile and cloud solutions

- Type of artefacts produced: This parameter involves the output of the activity or the event. It is considered as good practice if the artefacts produced during the educational robotic event are interesting and engaging; participants are interested to use the artefacts and to apply them in different domains of their lives.

\section{EVALUATION OF THE ACTIVITY}

The description of the activity provides information regarding methods and results of its evaluation, including the perspectives of the participants and the reflection of the teacher-instructor on aspects that might need improvement or are going to be changed in next implementations.

\section{SUSTAINABILITY}

- Cost of the activity: This dimension involves information regarding mainly costs of the material and organizational costs. It is considered a good practice if the activity requires materials or tools that are reasonably priced compared to other related activities. An example of a relatively cost-effective technology with good cost-to-quality ratio is the LEGO technology and as for a relevantly low initial investment the Arduino technology.

- Activity Financing: The activity - event is considered a good practice with respect to this dimension if it has a sustainable model for financing in midterm period, e.g. self-financing through fees, wide voluntary base, partnership with public organizations such as municipalities, schools or long term sponsorship partners.

- Activity Repetition: An activity - event is considered a good practice if it is performed sustainably for at least three subsequent periods in close cooperation with schools or other educational organizations. 
The information regarding this parameter involves mainly the sharing of activity related material (i.e. manuals, guidelines etc), in a way (i.e open access, structuring of information) that allows the activity - event to be replicated by other relevant stakeholders (teachers, )

\section{First version of activity plan}

In this section we present the first version of the activity plan template. The basic pedagogical theory underlying its design is constructionism where learning is connected to powerful ideas inherent in constructions with personal meaning for the students. We also identify a social dimension in the construction process and a specific learning attitude growing out of sharing, discussing and negotiating ideas. Another characteristic of this first version of the activity plan is that it designed to be adaptable to different learning settings: i.e. formal - non formal. The structure of the activity plan template is modular and the intention is to allow "selective exposure" of its elements to different stakeholders (the term selective exposure is borrowed from Blikstein [13] to describe the intentional hiding of some of the templates elements according to the involved settings or stakeholders). This first version presented here is informed by an analysis of the best practices identified and it is based on previous work on activity plan templates that aim at the integration of digital technologies in learning [14]

In this section we present the main elements of the structure of Activity plan template (the full description is provided in Appendix A) involve: the description of the scenario with reference to the different domains involved, different types of objectives, duration and necessary material; the next section involves contextual information (regarding, space, participants etc); the third section focuses on issues regarding the social orchestration of the activity; the third section refers to the teaching and learning procedures and it is the field where the influence of the pedagogical theory is mostly demonstrated; Section 5 focuses on possible student constructions; section 6 provides a description of the sequencing and the focus of activities and section 7 is devoted to evaluation.

\section{Conclusion}

In this paper we discussed the role of activity plan templates as mediating artefacts in harnessing the potential of educational Robotics for learning and in addressing the issue of fragmentation in the domain. The concept of mediating artefact was adopted here to describe a generic learning design instrument that is constructed taking into account a) a specific pedagogical theory and b) the particularities of robotics as technologies. The activity plan template is an abstraction of what we have identified as essential and transferrable elements of learning with robotics. The work reported here is in progress, thus the activity plan template we present here is going to be evaluated in practice by teachers who will use it to create their own activity plans and by researchers and students during the implementation of these plans in practice. 
Feedback generated from this process will be used to inform the activity plan template so as a) to have a level of abstraction that it will make it adaptable to different settings and b)to have a level of detail that will demonstrate the influence of a specific pedagogical approach and will address the particularities of Robotics.

Acknowledgments. The project has received funding from the European Union's Horizon 2020 research and innovation program under grant agreement No. 665972. Project Educational Robotics for STEM: ER4STEM

\section{References}

1. Alimisis, D.: Robotic technologies as vehicles of new ways of thinking, about constructivist teaching and learning: the TERECoP Project. IEEE Robot. Autom. Mag. 16, 21-23 (2009).

2. Miller, M.: Mobile Building Blocks 2014: Mobile Cores. PC Mag. (2014).

3. Papert, S.: Mindstorms: Children, computers, and powerful ideas. Basic Books, Inc. (1980).

4. Simon, H.A.: The sciences of the artificial. MIT Press, Cambridge, MA (1969).

5. Mor, Y., Winters, N.: Design approaches in technology-enhanced learning. Interact. Learn. Environ. 15, 61-75 (2007).

6. The Design-Based Research Collective: Design-based research: An emerging paradigm for educational inquiry. Educ. Res. 5-8 (2003).

7. Trouche, L.: Managing the complexity of human/machine interactions in computerized learning environments: Guiding students' command process through instrumental orchestrations. Int. J. Comput. Math. Learn. 9, 281-307 (2004).

8. Gueudet, G., Trouche, L.: Towards new documentation systems for mathematics teachers? Educ. Stud. Math. 71, 199-218 (2009).

9. Verillon, P., Rabardel, P.: Cognition and artifacts: a contribution to the study of though in relation to instrumented activity. Eur. J. Psychol. Educ. 10, 77-101 (1995).

10. Pepin, B., Gueudet, G., Trouche, L.: Re-sourcing teachers' work and interactions: a collective perspective on resources, their use and transformation. ZDM. 45, 929-943 (2013).

11. Kynigos, C., Kalogeria, E.: Boundary crossing through in-service online mathematics teacher education: the case of scenarios and half-baked microworlds. ZDM. 1-13 (2012).

12. Conole, G.: The role of mediating artefacts in learning design. Handb. Res. Learn. Des. Learn. Objects Issues Appl. Technol. 108-208 (2008).

13. Blikstein, P.: Computationally Enhanced Toolkits for Children: Historical Review and a Framework for Future Design. Found. Trends ${ }^{\circledR}$ Human-Computer Interact. 9, 1-68 (2015).

14. Yiannoutsou, N., Kynigos, C.: Boundary Objects in Educational Design Research: designing an intervention for learning how to learn in collectives with technologies that support collaboration and exploratory learning. In: Plomp, T. and Nieveen, N. (eds.) Educational Design Research: Introduction and Illustrative Cases. pp. 357 - 379. SLO, Netherlands Institute for Curriculum Development, Enschede, The Netherlands (2013).

\section{Appendix A.}

\section{TITLE: of the activity as it is mediated to students}

\section{Author: teacher, designer}




\section{Description of the scenario}

\subsection{Domain}

1.1.1. Primary domain: e.g. Electrical engineering, Robotics, choose ONE category
1.1.1.1. Science
1.1.1.2. Technology
1.1.1.3. Business

1.1.1.4. Engineering (tick)

\subsubsection{Arts}

1.1.1.6. Mathematics

1.1.2. Contextual (peripheral) domain: e.g. Art, Biology \& Mathematics, also give a rating of the level of emphasis on concepts from each of these domains

1.1.2.1. Science $(0-10)$

1.1.2.2. Technology $(0-10)$

1.1.2.3. Business $(0-10)$

1.1.2.4. Engineering (0-10)

1.1.2.5. Arts $(0-10)$

1.1.2.6. Mathematics $(0-10)$

\subsection{Objectives}

1.2.1. Subject related: For example: Study the angle and position of all materials (servo motors, circuits, sensors), as well as the construction of the legs in order for the insect to be autonomous and move correctly.

1.2.2. Technology use related: For example: Programming of Arduino

1.2.3. Social and action related: For example: Improve collaborative skills, take roles within groups

1.2.4. Argumentation and fostering of maker culture: for example practice making conjectures about how the robot will react to external stimuli based on the program given

\subsection{Time}

1.3.1. Duration: 5 weeks

1.3.2. Schedule: 2 hours per week 


\subsection{Materials and Artifacts}

1.4.1. Digital artifact: For example: programming language, visual interface, robot simulation

1.4.2. Robotic artifact: the technology and the robot form, e.g. 'an insect', 'a car' ...

1.4.3. Student's workbook and manual: use a manual with step-by-step instructions for the electronic part and programming part too

1.4.4. Teacher's instruction book and manual: teacher's notes with a template of e.g. three incisive stages and five steps for the first two stages.

\section{Space and Student Info}

\subsection{Students Info (Target Audience)}

2.1.1. Sex and Age: For example: boys \& girls, 17 years old

2.1.2. Required Prior knowledge: For example: little if any knowledge of Arduino but experts on the electronic part of it.

2.1.3. Nationality and cultural background: For example: 5 pupils are from Albania and 10 from Greece

2.1.4. Social status and social environment: underpriviledged area, mainstream public school, elite private school

2.1.5. Special needs and abilities: For example: ADD, dyslexia, Soc. Em. Behavior Disorders, gifted, other

\subsection{Space Info}

2.2.1. Organizational and cultural context: For example: in school at the technology laboratory, during project time in after school established voluntary club activity

2.2.2. Physical characteristics: indoors, floor, ...

\section{Social Orchestration}

\subsection{Population}

3.1.1. Students: 15

3.1.2. Tutors: 2

\subsection{Grouping}

3.2.1. Setting: students in a normal classroom, around light mobile tables, looking at the blackboard, in small groups.

3.2.2. Grouping criteria: mixed ability, mixed gender 


\subsection{Kinds of Interaction during the activity (emphasis)}

3.3.1. Actions: exchange ideas, dialogue, negotiation, debate, ..

3.3.2. Relationships: collaborative, competitive

3.3.3. Roles in the group: constant roles, pre-defined roles, emergent roles

3.3.4. Support by the tutor(s): support, intervene, self-regulatory

\section{Teaching and Learning Procedures}

\subsection{Teacher's role}

4.1.1. Teacher' function: (what is the teacher doing?) mentor, consultant and researcher, lecturer

\subsection{Teaching methods}

4.2.1. Teacher's approaches: For example: demonstrate, engage by example,..

\subsection{Student activity processes}

4.3.1. Students' function: action, writing, observing, creating, ..

\subsection{Student learning processes}

4.4.1. Designed Conflicts and misconceptions: do the activity designers wish to bring students in conflict with mistaken conception they might have which are documented in ed. research? Which ones? what kind of conflict?

4.4.2. learning processes emphasised: For example: emphasis on studying robot behaviour as a result of the program the students gave (i.e. use behaviour as feedback on programming)

4.4.3. Expected relevance of alternative knowledge (which): For example: biology and especially the structure of an insect's body

\section{Student productions}

\subsection{Artifacts - robots}

5.1.1 assignment: (For whom is the robot? What tasks shall the robot perform?) entertain, bring stuff, call help, vacuum clean,...

5.2.2 interaction: (How to communicate with the robot?) speech, gesture, mind control, app,...

5.2.3 morphology: (How does the robot look like? What material is it made of?) machine-like, zoomorphic, antropomorphic, cartoon-like,...

5.2.4 behavior: (What shall the robot behave like?) butler, friend, pet, protector, teacher,... 
5.2.3 parts: (What parts are needed?) electronics, software, mechanics, hull,...

\subsection{Programs - code}

5.2.1 Structure of code-commands:

5.2.2 Elements (e.g. iteration, selection, variables):

5.2.3 Conditionals (e.g. event handling):

5.3 Discussions - arguments (describe the activity emphasis on one or more of the following types of discussion)

5.3.1 descriptive - explanatory: description of a situation, a construct or an idea for others to understand and /or to implement

5.3.2 alternative: provision of solutions to problems, provision of alternatives if a dead end is reached

5.3.3 critical - objection: revision of other's constructs and ideas, identification of problems, challenge of ideas

5.3.4 contributory - extending: sharing of resources, provision of ideas towards improving an existing construct or initial idea

\section{Sequence and description of activities}

Here we describe how we expect the teaching and the learning process to evolve. We might use phases or activities for this description. A suggested structuring of activities might involve: construction phase involving hands on the robot, discussion of what has been achieved, problems and new ideas, construction of the robot behavior and in parallel exchange of ideas and help seeking

\section{Assessment Procedures ( for teacher reflection or student feedback)}

\subsection{Formative assessment}

\subsubsection{Pupil voice activities (Interviews with students, Questionnaire)}

\subsubsection{Observation notes}

\subsubsection{Peer assessment}

\subsection{Summative assessment}

7.2.1. Essays

7.2.2. $\underline{\text { Tests }}$

7.2.3. Student productions (code-robots-textual discussions)

\subsubsection{Mark sheet}

